\title{
Design and Application of Smart Metering System for Micro Grid
}

\author{
Ringo P K Lee \\ Powerpeg NSI Ltd. \\ Hong Kong, China
}

\author{
Loi Lei Lai, Chun Sing Lai* \\ State Grid Energy Research Institute \\ Beijing, China \\ 1.1.1ai@ieee.org,me10ccl@my.brunel.ac.uk
}

\begin{abstract}
This paper reports some design and requirements needed for applying smart meters to micro grid. Few practical applications will be used to demonstrate the benefit obtained with this approach. International standard used for communication will be included in the design. Good practice will be proposed.
\end{abstract}

Keywords-Smart meter, micro grid, standard

\section{INTRODUCTION}

The existing role of the metering facilities for the power grid are mainly used for the revenue meters, maximum demand meters, energy check meters, panel meters or multi-function digital meters, grid meters and power quality meters etc. They will mainly be classified into 2 types.

a) Smart Meters (SM): intelligent electronic smart meters for power grid measurement, with power, energy and optional basic power quality measurement, should be able to communicate with centralized software or to share data to third-party system [1].

b) High End Smart Meters (HESM): intelligent electronic smart meters for power grid or micro grid measurement, with power, energy and optional basic power quality measurement plus additional advanced power quality, reliability, fault analysis captures and programmable capability, should be able to communicate with centralized software or to share data with third-party system [2]

Future smart grid development will involve a lot of intelligent features, interactive response and integration such as advanced communication, self healing, gateway, logging, fault diagnostic, interactive demand response, control logic, synchronization and integration with protection devices, phase measurement unit, waveform and load flow monitoring etc. [3].

This paper reports some design and requirements needed for applying smart meters to micro grid. Few practical applications will be used to demonstrate the benefit obtained with this approach. International standard used for communication will be included in the design [4]. Good practice will be proposed.

\section{THE BENEFITS}

With the application of the micro grid smart meter (MGSM), the measurement and the logged data will provide

\footnotetext{
*Chun Sing Lai is now on leave from University of Oxford, UK
}

the benefits to micro grid consumers. Some of the benefits are described as below:

\section{A. User Profile}

Micro grid smart meters provide logged data of different user groups such as 15 minutes logging interval and the profile can be obtained to analyze the user patterns such as the turn on/off time of lighting and power, temperature and air quality, etc. With this pattern obtained, it is possible to trace the source of over usage of the energy consumption by standby computers and TV sets.

\section{B. Energy Efficiency}

Building services design consultant could compare the design and the actual usage of the facilities such as Air Handling Unit, to identify whether it is running efficiently.

\section{Benchmarking}

Property manager could compare the energy consumption from different users or analyze the energy efficiency of the equipment at different locations or floors. They can benchmark the equipment 'efficiency'.

\section{Energy cost allocation and analysis}

Energy reporting systems can help facility managers to generate the energy charts, they can identify the source of the high energy cost and identify the energy management opportunities for implementation consideration.

\section{E. Air-conditioning system control}

Micro grid smart meter should be integrated with chiller plant control system for better energy saving and optimization.

\section{F. Demand control}

Micro grid smart meters (MGSM) can monitor the tariff scheme and pre-program an energy saving control logic. They can provide signal to building management system for the execution of different type of control schedule programs based on utilities tariff scheme.

\section{G. Preventive maintenance}

Lack of maintenance for facilities such as chiller will reduce the efficiency of the equipment which causes a higher 
energy usage. Facility manager can consider the checking of the energy trend as one of the preventive measures.

\section{H. Power quality}

There are many electronic loads used in the world, they cause power quality problem. For example, a lot of dimming ballasts used for lighting control at the same time will cause harmonic problem. The AV equipment at the site will be affected owing to high harmonic voltage, or current distortion and neutral current. Installation of MGSM will help checking the power quality and identifying the source of the problem.

\section{Demand response}

Smart meters from power utilities can share data with energy consumers via wireless/wired communication media. Thus, the building management system can receive more interactive information such as peak demand forecast from power utilities and it can re-arrange the load shedding schedules to lower the peak accordingly.

\section{MEASUREMENT}

For a micro grid, the typical parameters can be classified into several major types.

\section{A. Typical real time $R M S$ value}

They are phase and line voltages, currents and total power factors and the concerned leakage currents.

\section{B. Delivered/received energy data in micro grid}

They are $\mathrm{kWh}, \mathrm{kVA}$ and $\mathrm{kVAr}$ with timestamp, event, vector, etc.

\section{Event data}

They are $\min / \max$ values with time stamp for the above items. Besides, the sag/swell voltage or set point current trigger from $1 / 2$ to a few cycles RMS calculation is recommended. Transient event trigger from $\mathrm{s}$ to $\mathrm{ms}$ are preferred if available. In some cases, On/Off/Trip and busbar temperature monitoring are also included in the critical event monitoring system.

\section{Power quality}

The total voltage and current harmonic distortion measurement is needed. Individual odd current harmonic measurement may be extended to $15 \mathrm{th}$. In general, the typical characteristic of current harmonic contents from the electronic loads of the building services are dominated by $3 \mathrm{rd}$, 5th, 7th, 11 th and 13th order owing to the power electronic circuit characteristic.

\section{E. Renewable energy systems}

$\mathrm{PV}$ and wind turbines distribution generations will need some more additional parameters such as PV array (DC) voltage to inverter; inverter $\mathrm{DC}$ voltage max and min; $\mathrm{AC}$ power rating; inverter nominal, max. and min. AC voltage output per phase; recording of daily, monthly and annual energy production; $\mathrm{kWh}$ generated from the $\mathrm{PV} /$ wind and the equivalent $\mathrm{CO} 2$; energy flow direction; power factor; power quality; load response; and $\mathrm{AC}$ and $\mathrm{DC}$ leakage.

\section{DESIGN AND REQUIREMENT ISSUES}

The design and requirements for different kinds of customers need to be considered.

\section{A. Retail consumers}

For the high rise residential buildings, consumer houses, small enterprises and commercial offices, the smart meters are individually provided by power utilities $[5,6]$. Those smart meters not only measure power and energy for utilities but they also provide the communication gateway features to pass the information from the network gateway of utilities' peak demand request to the consumers' appliances such as heating/ air-conditioning systems/ washing machines, etc. Alternatively, it can be done via external gateway or data concentrator via the Automatic Metering Infrastructure (AMI) wireless network and back to the utilities via Wide Area Network (WAN).

In this case, the consumers' houses may need In-home displays to view the daily usage and the schedule control setting of their home appliances. Those control and communication setting may be integrated into this unit or separated device depending on the design [7].

Referring to Figure 1 [3], communication between the smart meters/home appliances and the "network gateway" are formed by wireless network such as ZigBee or wired network for newly designed building. The "network gateway" may include some intelligent control features which work together with the command message from the utilities' control centre. From the data concentrator to the power utilities can be wireless/wired network via WAN such as DSL/3G/GPRS/PSTN modems, etc. Wireless ZigBee is a good approach since it allows data to be passed to the network gateway in more than 1 communication path. The wireless communications for networks gateways are recommended to be 2 separated licensed free frequencies. One of them is for the wireless ZigBee network to the consumers. Another one is for the communication between gateways to avoid the interference. The 3rd communication port can be used to connect to utilities' control centre via WAN as mentioned above, it is recommended to have a minimum of 2 physical dual redundancy communication channels with the same protocol to secure the communication with encryption such as AES128 bits.

\section{B. Bulk or large tariff industrial or commercial consumers}

Typically, they have a lot of high power consumption facilities. The design of the AMI may be applicable. However, the main differences are the In-home display and the control of home appliances. There may be a need to communicate with heating or air-conditioning or building management systems of the commercial consumers such as hotel or high rise commercial buildings with 1 single meter. For the industrial consumers, there may be a requirement to communicate with 


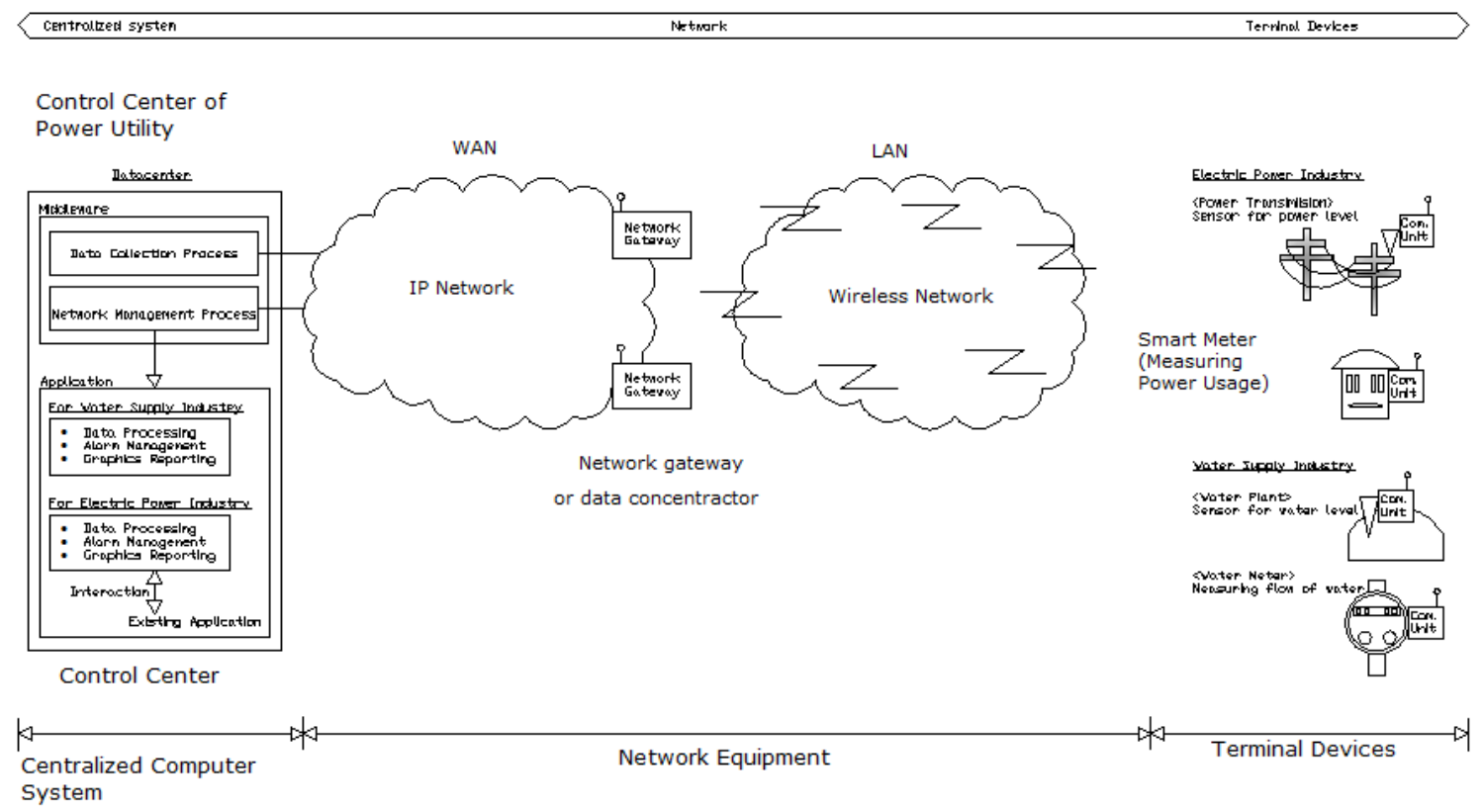

Fig. 1 Typical configuration for Automatic Metering Infrastructure (AMI)

proprietary protocol from the production line. The following interface methods are recommended.

I) Smart meters can establish a control interface $\mathrm{I} / \mathrm{O}$ unit with digital input/output to communicate with utilities' control centre for the peak demand response execution.

II) Smart meters can provide built-in or extended I/O unit to communicate with utilities' control centre for the peak demand response execution

In this case, the peak demand response signals can be passed to the bulk/large consumers for execution.

The communication between the control centre of the utility and the local management unit can be IEC 61850 via data concentrator with security control. There may be little PQ functions such as THD and sag/swell record [8]. Besides, ZigBee wireless module may be included to connect the wireless household appliances to receive the peak demand response signal and work together with power utilities interactively. Many manufacturers have produced some energy display and control panel to provide user interface and link up the MGSM together for Peak Demand control. The market is still under development.

Data sharing of smart meters is useful for peak demand response program for energy saving. It can be implemented by building management system and PLC, etc for commercial buildings.

For industrial sectors, PLC may be more common but the peak demand shedding may be done on other selective loads. The sharing protocol can be Modbus TCP or RTU etc open format. It depends on the availability and operation of the site.

High end smart meters (HESM) can be used in distributed generation for micro grid or main incoming feeders supplied by power utilities to perform more interaction such as energy conservation and peak demand response from power utilities if high end power quality features and programming are required.

The integration can be done via the utilities smart meters and interface with the building management systems. The typical methods can be as follows:

High level: open protocol such as IEC61850, Modbus RTU/TCP or BACNET, etc.

Low level: optical pulse, dry contact, pulse contact, analogue transducer, etc.

\section{System operation in power utilities application}

Typically, protection engineers would like to use one protection device to perform a single protection task rather than multiple tasks at the same time. It helps to improve the power system reliability and it is a good practice as well [9].

However, future smart grid will have more distributed renewable energy sources such as CHP generator, Plug-In Hybrid Electric Vehicle (PHEV) or energy storage power for peak demand response. They may affect the traditional power system reliability. To adopt more valuable information from smart meters and to cross check the precision of the protection, tripping time, voltage dip impact and transient system response from those renewable energy sources, etc. Protection engineers may consider the information sharing scheme from the smart meters as well. From the captured waveform, they can double check the protection performance and get the feedback from those newly installed renewable energy sources. Besides, engineers will adjust the setting if necessary, finally increase the system reliability and design a more reliable self-healing scheme. 
HESM is recommended to be $128 / 256$ samples per cycle at least. It will mainly be used for Power Grid network application with IEC 61850 protocol and share the same data to digital substation automation via IEC 61850. The other applications are the cross checking of system fault or events captured by other utility systems [10].

Smart meters (SM) are recommended for all other applications on power generation, transmission, distribution, energy storage and distributed generations. The IEC61850 should be included for peak demand response of data sharing to customers.

\section{Application Areas}

Design engineers need to know different applications for electrical circuits and some areas such as electrical distribution; data centre; plumbing \& drainage. With the proper installation of MGSM for micro grid, energy saving and peak demand management can be achieved more easily..

The following figures show some application examples.

a) User profile

Figure 2 shows the 7 days behavior of energy consumption of a commercial building. Differences from day to day operation can be carefully studied to identify the energy management opportunity. It indicates the exceed loading over the expected usage and the property manager may try to find out the reason to reduce the peak.

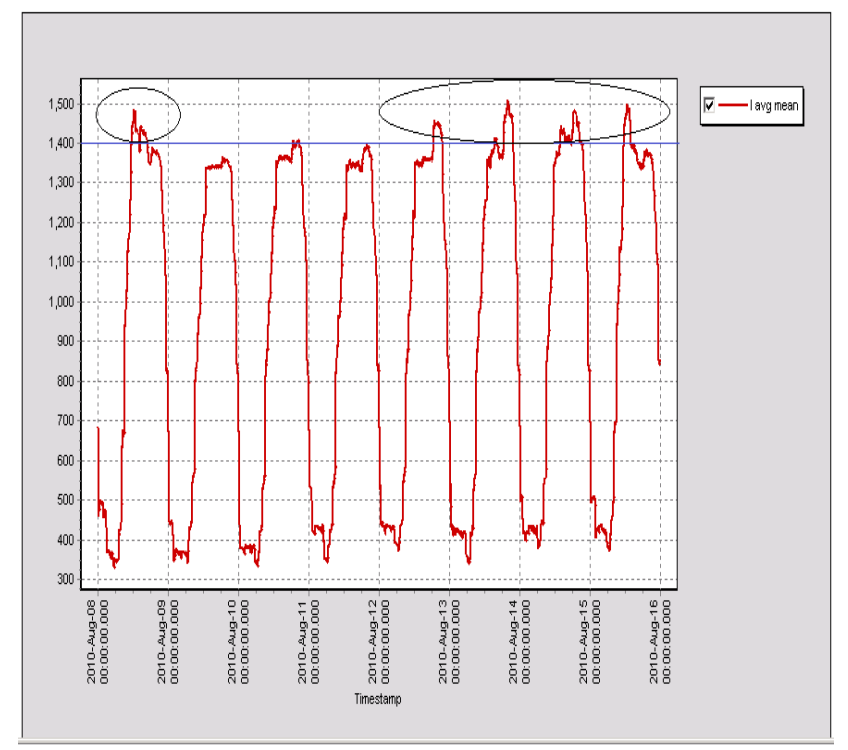

Fig. 2 Load profile for a week

b) Benchmarking

Figure 3 shows the energy usage of lighting, power and airconditioning of a government building from different floors which help to identify the most efficient electrical installation services from different locations.

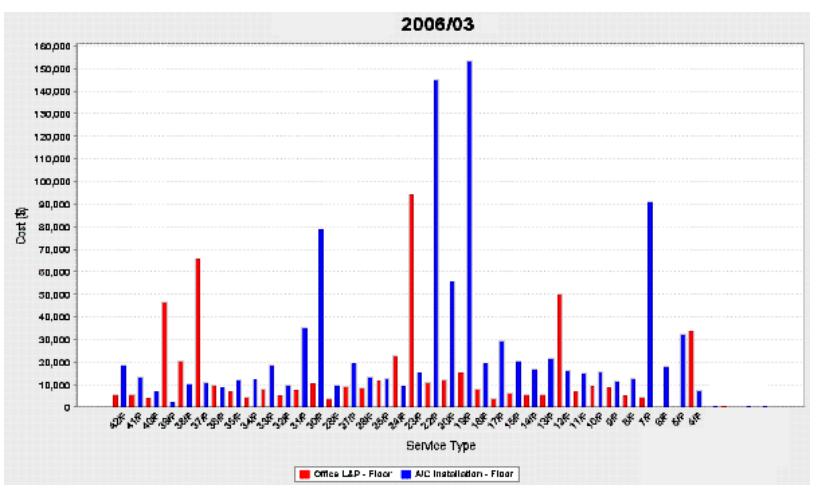

Fig. 3 Lighting and power usage are in red color and A/C usage in blue color [11]

c) Power quality and preventive maintenance

Capturing the event such as circuit trip, voltage sag/swell or harmonic can help to trace the source of the event happening. Fig. 4 shows the starting current from a motor machine. Fig. 5 shows the voltage dip more than $30 \%$ for 4 cycles from utility power supply. Fig. 6 shows circuit trip after voltage dip event from another incident. In this case, the pump control devices cannot ride-through the voltage dip caused the tripping. Engineers may install the ride-through device based on the event figure. Fig. 7 shows the PV generation profile of a small solar panel for 2 days from a shopping centre. It will reflect the true figures instead of the estimation. It was found that the generation will be consumed by the PV power inverter if it is too small and the overhead should be considered as well. By observation, the PV generation started from about 7:00am to 5:00pm. However, the July 31 has more energy generated but Aug 1 dropped a lot which may be caused from the weather. Since inverter with islanding protection is needed for PV system connected to utility grid. If the generated energy is too small, they will be consumed by the control and inverter equipment and waste the energy finally.

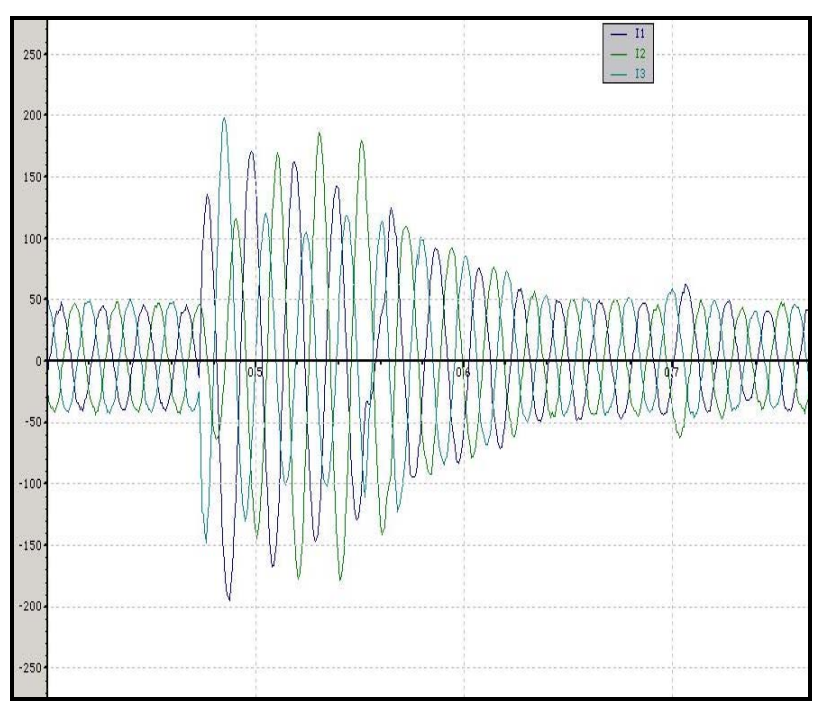

Fig. 4 Starting current from a motor 


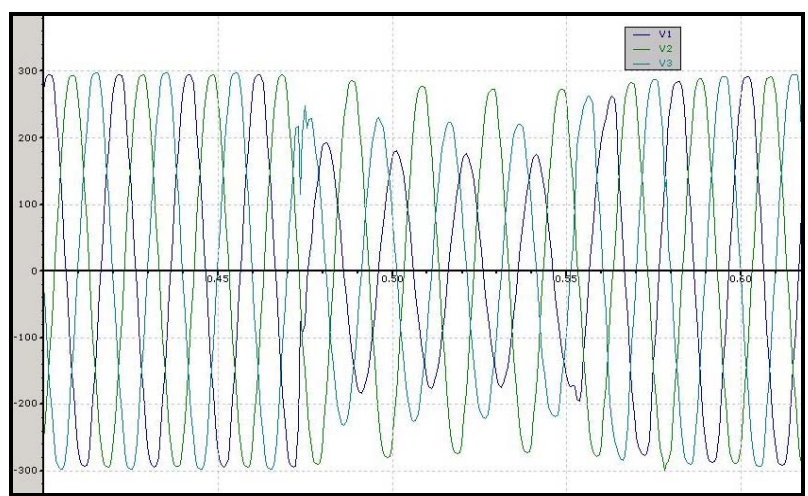

Fig. 5 Voltage dip capture for a motor

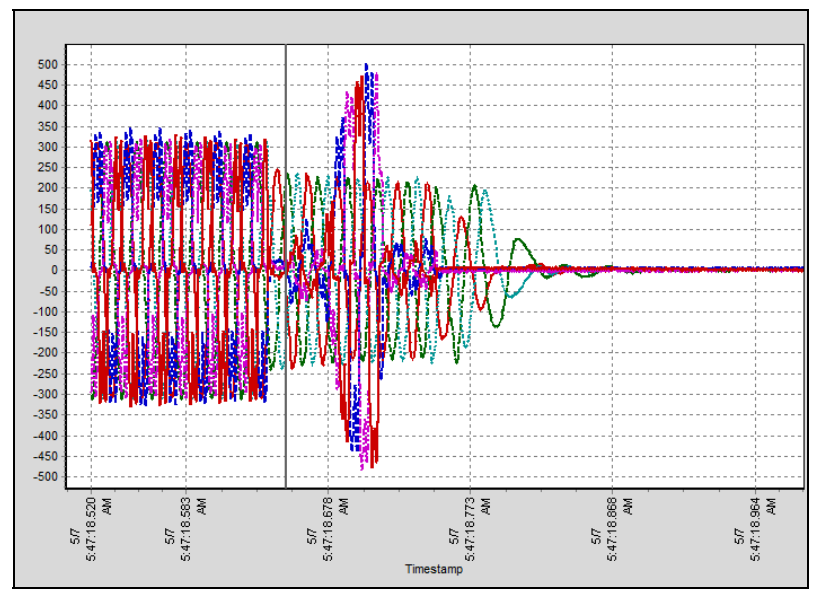

Fig. 6 Circuit trip after voltage dip

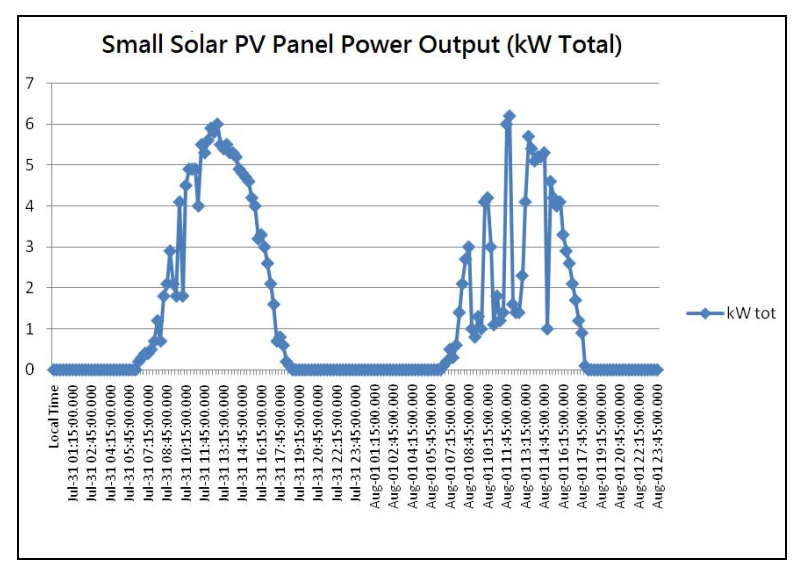

Fig. 7 Profile of a solar panel power output for 2 days

\section{CONCLUSIONS}

For the residential or small commercial consumers, they may not have micro grid smart meters. The energy monitoring can be achieved by the shared information from the smart meters (SM) provided by power utilities.

For the bulk or large tariff consumers such as large commercial, public utilities, hospitals, industrial sectors and shopping malls with distributed generation or energy storage, MGSM is provided by building owners/ properties management or energy consumers. The MGSM will be interfaced with building management system, industrial automation system, home automation system etc. for peak demand response, energy monitoring and saving. HESM may be used if advanced features are required as mentioned above. Of course, the SM or HESM from power utilities can be used if data sharing or peak demand response from power utility is needed.

For the power utilities, SM and HESM will be interfaced with SCADA system, protection \& control system, fault location, and recording systems etc. They will serve for power generation, distributed generation \& energy storage, transmission and distribution in HV level. The design purpose is different from that of the micro grid applications. Therefore, both of them have different application roles.

\section{REFERENCES}

[1] http://www.beama.org.uk/hottopics/Smart+Metering/defa ult.asp. Visited on 20 th of June, 2011

[2] http://en.wikipedia.org/wiki/Smart_meter. Visited on 16th of June, 2011

[3] http://www.fujitsu.com/global/solutions/smartgrid/smartn etwork/meterdata/ Smart Network Solution for Smart Grid from Fujitsu. Visited on 10th of May, 2011

[4] P2030.101 - Guide for Designing a Time Synchronization System for Power Substations, http://standards.ieee.org/develop/wg/Time Synchronizati on Design.html Visited on 10th July of 2013

[5] So A.T.P. and Chan W.L., Intelligent Building Systems, Kluwer Academic Press, April 1999.

[6] http://www.eia.gov/consumption/residential/. Visited on 19th of April, 2011

[7] http://www.Itron.com. Visited on 29th of April, 2011

[8] http://www.sandc.com/products/power-quality/purewavedstatcom.asp from S\&C. (Visited on 10th of May, 2011)

[9] http://products.schneider-electric.us/productsservices/products/power-management-products. (Visited on 7 th of June, 2011)

[10] http://www.rad.com/10/SHDSL-Ethernet-Modem-andMux/2933/ (Visited on 10th of May, 2011)

[11] Lee, P.K. and Lai, L.L., "Smart metering in micro-grid applications," IEEE PES 2009 Annual Meeting, July 2009, USA. 